Supplementary Information

\title{
Targeting small GTPases and their prenylation in diabetes mellitus
}

Edyta Gendaszewska-Darmach ${ }^{*}$, Malgorzata A. Garstka²*, Katarzyna M. Błażewska ${ }^{3 *}$

Institute of Molecular and Industrial Biotechnology, Faculty of Biotechnology and Food Sciences, Lodz University of Technology,

Stefanowskiego 4/10, 90-924 Lodz, Poland; edyta.gendaszewska-darmach@p.lodz.pl

${ }^{2}$ Core Research Laboratory, Department of Endocrinology, Department of Tumor and Immunology, Precision Medical Institute,

Western China Science and Technology Innovation Port, School of Medicine, the Second Affiliated Hospital of Xi'an Jiaotong,

DaMingGong, Jian Qiang Road, Wei Yang district, Xi'an 710016, China, m.garstka@xjtu.edu.cn

${ }^{3}$ Institute of Organic Chemistry, Faculty of Chemistry Lodz University of Technology, Zeromskiego St. 116, 90-924 Lodz, Poland; katarzyna.blazewska@p.lodz.pl

*Co-corresponding Authors

Content

1. Supplementary Figure S2-S6

2. Supplementary Table $1 \quad$ S7-S10

3. Supplementary Table $2 \quad$ S11-S13 


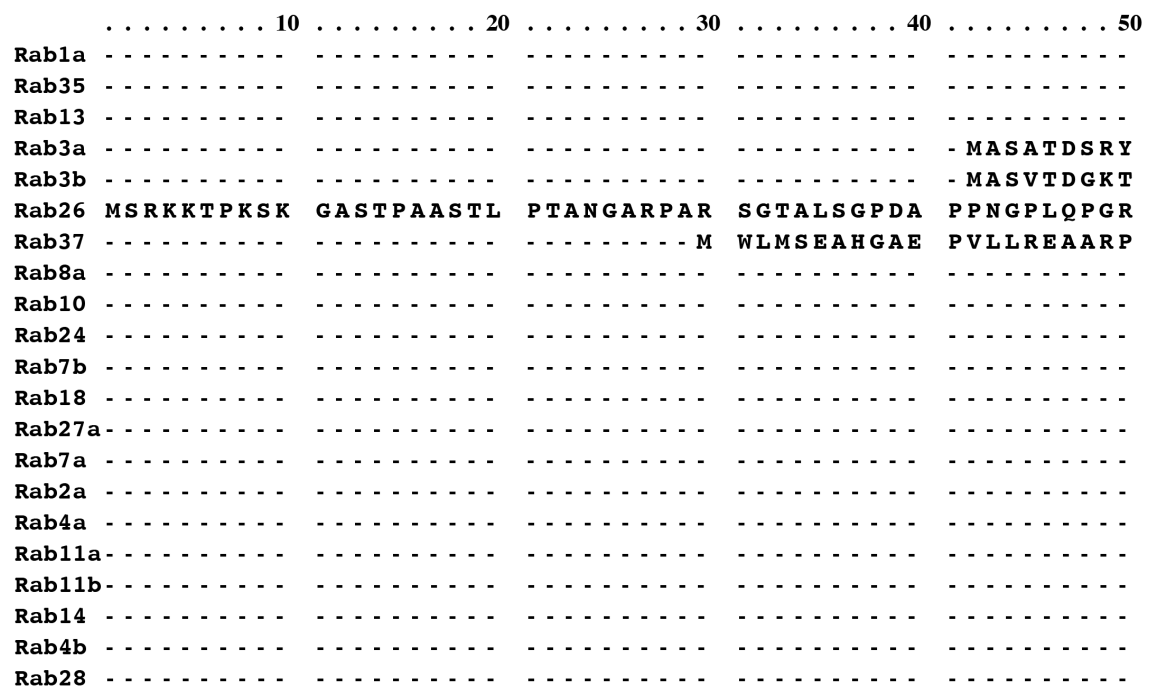

0 $\begin{array}{lllll}\text { - MSSMNPEY DYLFKLLLIG } & 7 \text { DSGVGKSCLL LRFADDTY - T ESYISTIGVD }\end{array}$ - - - MARDY DHLFKLLIIG DSGVGKSLL LRFADTF-S GSITIIGVD - - - MAKAY DHLFKLLLIG DSGVGKTCLI IRFAEDNF-N NTYISTIGID GQKESSDQNF DYMFKILIIG NSSVGKTSFL FRYADDSF-T PAFVSTVGID GVKDASDQNF DYMFKLLIIG NSSVGKTSFL FRYADTF-T PAFVSTVGID PSLGGGVDF DVAFKVMLVG DSGVGKTCLL VRFKDGAFLA GTFISTVGID

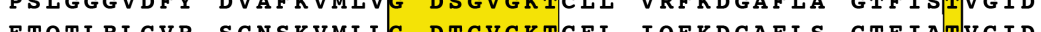
FTQTLRLCVP SGNSKVMLLG DTGVGKTCFL IQFKDGAFLS GTFIATVGID --.- MAKTY DYLFKLLLIG DSGVGKTCVL FRFSEDAF-N STFISTIGID - - - MAKKTY DLLFKLLLIG DSGVGKTCVL FRFSDDAF-N TTFISTIGID -.. - MS-GQ RVDVKVVMLG KEYVGKTSLV ERYVHDRFLV GPYQNTIGAA - . - . MNPRK KVDLKLIIVG AIGVGKTSLL HQYVHKTF-Y EEYQTTLGAS - - - MDEDV LTTLKILIIG ESGVGKSSLL LRFTDDTF-D PELAATIGVD -. - MSDGDY DYLIKFLALG DSGVGKTSVL YQYTDGKF-N SKFITTVGID -. - - MTSRK KVLLKVIILG DSGVGKTSLM NQYVNKKF-S NOYKATIGAD -. . . - MAY AYLFKYIIIG DTGVGKSCLL LQFTDKRF- $Q$ PVHDITIGVE MSQTAMSETY DFLFKFLIG NAGTGKSCLL HQFIEKKF-K DDSNTIGVE MGTR - - DDEY DYLFKVVLIG DSGVGKSNLL SRFTRNEF-N LESKSTIGVE MGTR- - DDEY DYLFKVVLIG DSGVGKSNLL SRFTRNEF-N LESKSTIGVE MATA - PYNY SYIFKYIIIG DMGVGKSCLI HQFTEKKF-M ADCPHTIGVE $\begin{array}{llllll}\text { M - - - - AETY DFLFKLLIG } & \text { SAGTGKSCLL } & \text { HQFIENKF-K } & \text { QDSNHTIGVE } \\ \text { MSDS-EESO } & \text { DROLKIVVLG } & \text { DGASGKTST } & \text { TCFAOETF-G } & \text { KOYKOTIGLD }\end{array}$

Switch II
110 . Rab1a FKIRTIELD Rab35 FKIRTVEINRab13 FKIRTVDIE Rab13 FKIRIVDIE Rab3a FKVKTIYRN Rab3b PKVKIVYRH Rab26 PRIKVLDVD Rab37 FRNKVVTVD Rab8a FKIRTIELD Rab10 FKIKTVELQ Rab24 FVAK VMS VG. Rab7b I LSKI I ILG Rab18 FKVKTISVD. Rab27aFRER Rab27a PREKR V V Y R Rab7a FLIKEVMVD Rab2a FGARMITID Rab4a FGSKI INVG Rab1laFATRSIQVD Rab11bFATRS I QVD Rab14 FGTRIIEVS Rab4b FGSRVVNVG Rab28 FFLRRITLP.
130 . K- TIKLOIW -

- . . . .

- G E- KVKLQIW-

$K-K I K L Q V W$ -

$K-R I K L Q I W-$

K-RVKLQIW

V-KVKLQMW

V-RVKLQIW

$K$ - RIKLQIW

$K-K I K L Q I W$

D T- TLKLQIW-

N-KAKLAIWV

SGPDGATGRG O-RIHLQLW

R- LVTMQIW-

$K-Q I K L Q I W$ -

$K-Y V K L Q I W$

$K$ - TIKAQIW -

$K-T I K A Q I W$

$Q-K I K L Q I W$

$K-T V K L Q I W$
. . . . . 140. . . . . 150 $\ldots \ldots$ $\ldots \ldots \ldots$ $-\ldots \ldots$ $\ldots \ldots \ldots$ [. -

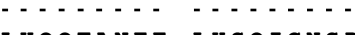
$\ldots \ldots$ $\ldots-1, \ldots$ ..... .
50 G3

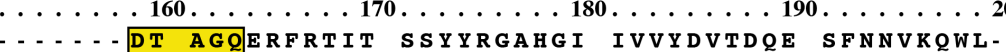

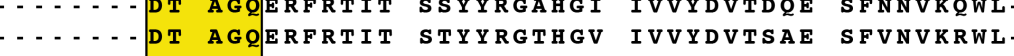

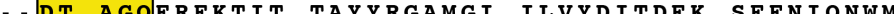
.... . DT AGQERYRTIT TAYYRGAMGF ILMYDITNEE SFNAVQDWS .... DT AGOERYRTIT TAYYRGAMGF ILMYDITNEE SFNAVQDWA

..... . DT AGOERFRST HAYYRDAHAL LLLYDVTNKA SFDIQAWL

... DT AGQERFRST HAYYRDAQAL LLLYDITNKS SFDIRAWL

D AGOERFRTIT TAYYRGAMGI MLVYDITNEK SFDNIRNWI

DT AGQERFHTIT TSYYRGAMGI MLVYDITNGK SFENISKWL

DT AGSERYEAMS RIYYRGAKAA IVCYDLTDSS SFERAKFWV

DT GGOERFRSMV STFYKGSDGC ILAFDVTDLE SFEALDIWR

LKWAMWOYDT AGOFRFRTIT PSYYRGAOGV ILVYDVTRRD TFVKIDNWLAUG LLFDLTMR SFLUVRWW -

... DT AGQERFQSLG VAFYRGADCC VLVFDVTAPN TFKTLDSWR

..... DT AGOESFRSIT RSYYRGAAGA LIVYDITRRD TFNHLTTWL

DT AGOERFRSVT RSYYRGAAGA LIVYDITSRE TYNALTNWL

DT AGQERFRST RSYYRGAAGA LLVYDITSRE TYNALTNWL

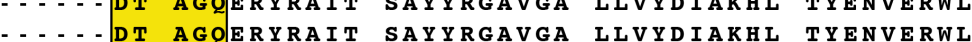

$\begin{array}{llll}- & \text { DT AGQERFRAT RSYYRGAAGA LMVYDITRRS TYNHLSSWL }\end{array}$

.... D T AGQERFRST RSYYRGAAGA LLVYDITSRE TYNSLAAWL

. . . . . DI GGOTIGGKML DKYIYGAQGV LLVYDITNYQ SFENLEDYT 
$\ldots \ldots 210 \ldots \ldots 220 \ldots .64$ Rab1a - - QEIDRYAS - - EN - VNKL LVGNKCDIT- - - TKKVVDY TTAKEFADSL Rab35 - - HEINQNC - - - - DD - VCRI LVGNKNDDP - - - ERKVVET EDAYKFAGQM Rab13 - - KSIKENAS - - A A - VERL LLGNKCDME - - - AKRKVQK EQADKLAREH Rab3a - - TQIKTYSW - - DN-AQVL LVGNKCDME - - - DERVVSS ERGRQLADHL Rab3b - - TQIKTYSW - - DN - AQVI LVGNKCDME - - - EERVVPT EKGQLLAEQL Rab26 - - TEIHEYAQ - - - HD - VALM LLGNKVDSA - - - - HERVVKR EDGEKLAKEY Rab37 - - TEIHEYAQ - - R RD - VVIM LLGNKADMS - - - S SERVIRS EDGETLAREY Rab8 - RNIERHAS - . AD VEKM ILGMKCDVN - DKROVSK ERGEKLALDY Rab10 - . RNIDEHAN - . ED - VERM LLGNKCDMD - . - DKRVVPK GKGEQIAREH Rab10 - - RUIDEHAN - - -ED-VERM LLGNKCDMD- - - - DKRVVPK GKGEQIAREH Rab24 - - KEL-RSLE - - EEG-CQIY LCGTKSDLLE EDRRRRRVDF HDVQDYADNI Rab7b - - GDVLAKIV PMEQS- YPMV LLGNKIDLA - - - - DRKVPQ EVAQGWCREK Rab18 - - NELETYCT - - RNDIVNM LVGNKIDE - - - NREVDR NEGLKFARKH Rab27a - - SQLQMHAY - - C CENPDIV LCGNKSDLE - - - DQRVVKE EEAIALAEKY Rab7a - - DEFLIOAS PRDPENFPFV VLGNKIDLE- - - NROVATK RAOAWCYSKN Rab2a - - EDAROHS - . . NSNMVIM IIGNKSDIE - . . SRREVKK EEGEAFAREH

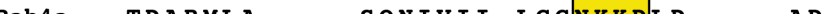
Raba - TDARMLA - - S SQNIVII LCGNKRDLD- - - - ADREVTF LEASRFAQEN

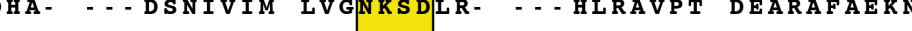
Rab11b- - KELRDHA - - - D DSNIVIM LVGNKSDLR- - . HLRA Rab14 - - TDARNLT - - - NPNTVII LIGNKADIE - - - AQRDVTY EEAKQFAEEN Rab4b - - TDARTLA - - - S SPNIVVI LCGNKKDLD - - - PEREVTF LEASRFAQEN Rab28 VVKKVSEES - - - E ETQPLVA LVGNKIDLE- - . - HMRTIKP EKHLRFCQEN

\begin{tabular}{|c|c|c|c|c|}
\hline גד & NATNVEQSFM & I & G P GA $\cdots \cdots$ & $A E$ \\
\hline $\mathbf{A K}]$ & ENVNVEEMFN & CITELVLRAK & KDNL- - - & - AKQQQQ \\
\hline & SMNVDEAFS & SLARDILLKS & G G R R - & - SGNGNKP \\
\hline$F$ & NINVKQTFE & RLVDVICEKM & SESL - - DT & ADPAVTGAKQ \\
\hline $\mathbf{A K} \mid$ & NISVRQAFE & RLVDA I C DKM & SDSL- & T D P SMLGSSK \\
\hline $\mathbf{K}$ & TGLNVDLAFT & A I AKELKQRS & MKAP - - - - & - - - SEPR \\
\hline FLE & TGMNVELAFL & A I AKELKYRA & Q $\mathrm{A}-$ & - - - DEPS \\
\hline$F$ & SNAF F & L ARD I K A K M & $-\cdots$ & GN S P \\
\hline I RFFETSAK & KAFL & LAEDILRKT & PVKE & EN V \\
\hline QLFE & GQS V DELF $Q$ & K V A E D Y V S VA & AFQV $\ldots$ & - - - MTEDK \\
\hline Y F & NDINVVQAFE & MLASRALSRY & QSIL $\ldots$ & - . E ENHL \\
\hline & $\mathbf{F E}$ & $T P$ & $\checkmark$ & N K \\
\hline 1 & A I E & RM & DK & VRS \\
\hline S A K & EA I NVEQAFQ & TIARNALKQE & TEVE- & - - LYNEFPE \\
\hline I F M I & TASNVEEAFI & NTAKEIYEKI & [NNE & A NG I K I G P QH \\
\hline & EAFV & $\mathrm{KI}$ & E R & 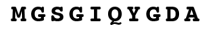 \\
\hline 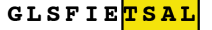 & AFQ & E I Y R I V & D R R & P S N N \\
\hline $\mathbf{L} \mathbf{S F}$ & D S T & E I Y R I V & SQKO & AAHDESPGNN \\
\hline & NVEDAFL & K K I Y & L N & TOH K P \\
\hline & N VEEA & T I L N K I & GEL - & I \\
\hline & & & & \\
\hline
\end{tabular}

$\ldots \ldots \ldots 310 \ldots \ldots \ldots 320 \ldots \ldots$

Rabla NVKIOSTPVK O-SG.- GGC - C -

Rab35 NDVVKLTKNS K-RK- - KRC - C. -

Rab13 STDLKTCDK- K - NT - - NKC - SLG-

Rab3a GPQLSDQQVP P - HQ- - - DCA - C. -

Rab3b NTRLSDTPPL L - OO - . NCS - C. .

Rab26 FRIHDYVKRE G-RG- - ASC - CRP

Rab37 FQIRDYVESQ K-KR- - SSC - CSFM

Rab8a GVKITPDQQK R-SS- - FFR - CVLI

Rab10 DISSGGGVT- G- WK - - - SKC - C.- -

Rab24 GVDLGQKPN- P- YF- - Y Y C - CHH

Rab7b TESIKLSPD- Q-SR- - - SRC - C- -

Rab18 GVKLSHREEG Q- GGGACGGY - CSVL

Rab27aNGHASTDOLS E-EKE- - KGA - CGC.

Rab7 P PILDKNDRA K - AS- - AES

Rab7a PIKLDKMDRA K ASS-AES - CSC

Rab2a AATNATHAGN QGGQQAGGGC - C-.

Rab4a ALRQLRSPRR AQAPNAQECG - C-

Rab1laVVPIHVPPTT E- - NKPKVQC - CQNI

Rab11bVVDISVPPTT DGQKPNKLQC - CQNL

Rab14 SAPQGGRLTS EPQPQREGCG - C.-

Rab4b ASLRQLRQPR SAQAVAPQPC GC-. -

Rab28 IVNYNQEPMS RTVNPPRSSM - CAVQ

Prenylation 
B

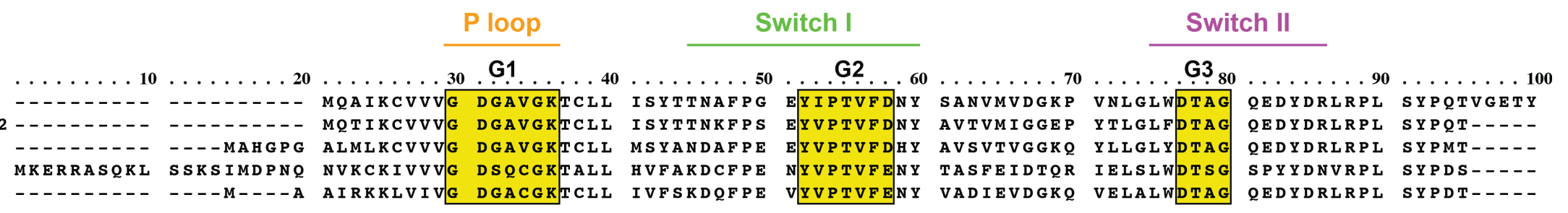

RhoA

G4

Rac1 GKDITSRGKD KPIADVFLIC FSLVSPASFE NVRAKWYPEV RHHCPNTPII LVGTKLDLRD DKDTIEKLKE KKLTPITYPQ GLAMAKEIGA VKYLECSALCdC42 --- - - - DVFLVC FSVVSPSSFE NVKEKWVPEI THHCPKTPFL LVGTQIDIRD DPSTIEKLAK NKQKPITPET AEKLARDLKA VKYVECSALTC10 -

RND3 - - - - - DAVLIC FDISRPETLD SVLKKWKGEI OEFCPNTKML LVGCKSDLRT DVSTLVELSN HROTPVSYDO GANMAKOIGA ATYIECSALO RhOA - - - - - - DVILMC FSIDSPDSLE NIPEKWTPEV KHFCPNVPII LVGNKDLRN DEHTRRLAK MKQEPVKPEE GRDMANRIGA FGMECSAK-

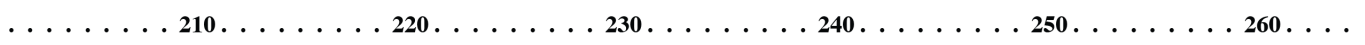

RaC1 TQRGLKTVFD EAIRAVLCPP PVKKRKRK -

CdC42 TOKGLKNVFD EAILAALEPP EPKK-SRR-- - - - - -

TC10 TQKGLKTVFD EAIIAILTPK KHTVKKRIGS RCIN-_-_.

$\begin{array}{llllllll}\text { RND3 } & \text { SENSVRDIFH } & \text { VATLACVNKT } & \text { NKNVKRNKSQ } & \text { RATKRISHMP } & \text { SRPELSAVAT } & \text { DLRKDKAKSC } & \text { TVM - } \\ \text { RhOA } & \text { TKDGVREVFE } & \text { MATRAALOAR } & \text { RGKKKSG-- } & -\end{array}$

Prenylation 


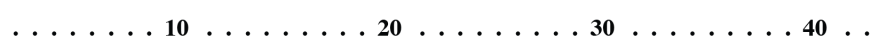

RalA - -

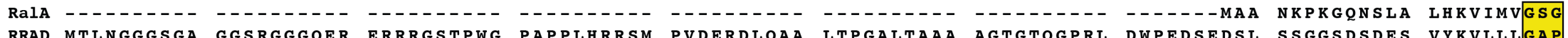
HRas NRas KRAs Rap1a-10 Rap1b-1-_-_ -

Ploop Switch I

Switch II

G1 $110 \ldots$ G2 $130 \ldots \ldots$

G Rala RRAD GVGKSALARI FGGVED-GPE AEAAG HRAS GVGKSALTIQ LIQNHFVDEY DPTIEDSYRK QVVIDGETCL LDILDTAGQE EYSAMRDQYM RTGEGFLCVF AINATSFED IHQYRQIKR VKDSDDVPMV NRas GVGKSALTIQ LIQNHFVDEY DPTIEDSYRK QVVIDGETCL LDILDTAGQE EYSAMRDQYM RTGEGFLCVF AinNSKFAD INLYReIKR VRDSDDVMV

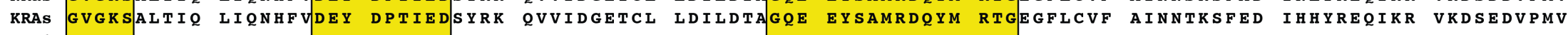
Rap1aGVGKSALTV FVQGIFVEKY DPTIEDSYRK QVEVDCQQCM LEILDTAGTE QFTAMRDLYM KNGQGFALY SITAQSTFND LQDLREILR VKDTEDVPMI

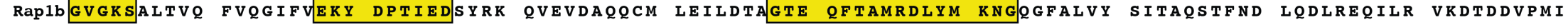

\section{G4}

$$
\text { G5 }
$$

RalA

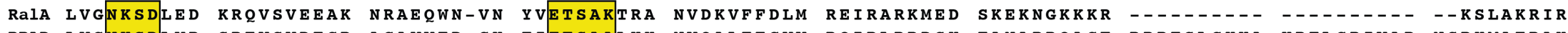
RRAD LVGNKSDIVR SREVSVDEGR ACAVVFD-CK FIETSAALHA NVQALFEGVV RQIRLRRDSK EANARRQAT RRRESLGKKA KRFLGRIVAR NSRKMAFRAK HRAS LVGNKCDLAA -RTVESRQAQ DLARSYG-IP YIETSAKTRQ GVEDAFYTLV REIRQHKLRK LNPPD- - - - - - - - - - - - - - - - - - - ESGPGCMS NRAS LVGNKCDLPT -RTVDTKOAH ELAKSYG-IP FIETSAKTRO GVEDAFYTLV REIROYRMKK LNSSD-_._. KRAS LVGTRDIPS RTVDTKQAQ DLARSVG IP FI KRAS LVGUKCDLPS -RTVDTKQAQ DLARSYG-IP FI TSAKIRQ RVEDAFYTLV REIRQYRLKK ISKEE--$\begin{array}{lllllllll}\text { Rapla LVGNKCDIED } & \text { ERVVGKEQGQ } & \text { NLARQWCNCA } & \text { FLESSAKSKI } & \text { NVNEIFYDLV RQINRKTPVE } & \text { KKKPK- } \\ \text { Rap1bLVGNKCDIED } & \text { ERVVGKEQGQ } & \text { NLARQWNNCA } & \text { FLESSAKSKI } & \text { NVNEIFYDLV } & \text { RQINRKTPVP } & \text { GKARK- } & & \end{array}$ $\ldots \ldots . \ldots 310$

RalA ERCC----IL

RRAD SKSCHDLSVL
HRAS CKCV----LS

NRas L PCV----VM

KRAs K KCI - - - IM

Rap1a - S CL - - - - L L

Rap1b - S C Q - - - - L L

Prenylation 
Supplementary Figure 1 corresponding to Figure 1. Amino acid sequence alignment of human GTPases involved in diabetes and insulin resistance

Protein sequences were downloaded from String: functional proteins association network. Alignment of Rab (A), Rho (B) and Ras (C) proteins was performed using Praline (developed in the Centre for Integrative Bioinformatics Vrije Universteit Amsterdam). Important regions are indicated: the P loop (orange), switch I (green) and switch II (magenta), the nucleotide-binding G-motifs (yellow, G1-G5) and the C-terminal cysteine important for prenylation (cyan). Numbers at the top indicate the residue number. 
Supplementary Table 1 corresponding to Figure 1. List of crystal structures of small GTPases playing a role in diabetes.

\begin{tabular}{|c|c|c|c|c|c|}
\hline GTPase & GDP-bound & $\begin{array}{l}\text { GTP\&_ } \\
\text { bound }\end{array}$ & $\begin{array}{c}\text { In complex } \\
\text { with effector }\end{array}$ & $\begin{array}{l}\text { In complex with } \\
\text { GEF }\end{array}$ & $\begin{array}{c}\text { In complex } \\
\text { with GAD }\end{array}$ \\
\hline \multicolumn{6}{|c|}{ Rab GTPases } \\
\hline Rabla & $2 \mathrm{FOL}$ & ND & $\mathrm{ND}^{\wedge}$ & $\mathrm{ND}^{\wedge}$ & $\mathrm{ND}^{\wedge}$ \\
\hline Rab2a & ND & ND & ND & ND & ND \\
\hline Rab3a & ND & $3 \mathrm{RAB}$ & ND & ND & ND \\
\hline Rab3b & $3 \mathrm{DZ} 8$ & ND & ND & ND & ND \\
\hline Rab4a & 2BMD & $\begin{array}{l}\text { 1YU9 } \\
\text { 2BME }\end{array}$ & ND & ND & ND \\
\hline Rab4b & $2 \mathrm{O} 52$ & ND & ND & ND & ND \\
\hline Rab5a & $\begin{array}{l}1 \mathrm{TU} 4 \\
2 \mathrm{BMD}\end{array}$ & $1 \mathrm{~N} 6 \mathrm{H}$ & $\mathrm{ND}^{\wedge}$ & $\mathrm{ND}^{\wedge}$ & ND \\
\hline Rab7 & 1VG1 & $\begin{array}{c}1 \mathrm{~T} 91 \\
\mathbf{1 V G 8}\end{array}$ & 1YHN (RILP) & ND & ND \\
\hline Rab8a & $\begin{array}{l}4 \mathrm{LHV} \\
6 \mathrm{STF}\end{array}$ & $\begin{array}{l}\text { 4LHW } \\
6 \mathrm{STG}\end{array}$ & $\mathrm{ND}^{\wedge}$ & $\mathrm{ND}^{\wedge}$ & $\mathrm{ND}^{\wedge}$ \\
\hline Rab10 & ND & ND & $\mathrm{ND}^{\wedge}$ & ND & ND \\
\hline Rab11a & $\begin{array}{c}1 \mathrm{OIV} \\
1 \mathrm{OIX} \text { (and } \\
\mathrm{Pi} \text { ) } \\
6 \mathrm{IY} 1\end{array}$ & $\begin{array}{l}1 \mathrm{OIW} \\
1 \mathrm{YZK}\end{array}$ & $\mathrm{ND}^{\wedge}$ & $\mathrm{ND}^{\wedge}$ & ND \\
\hline
\end{tabular}




\begin{tabular}{|c|c|c|c|c|c|}
\hline Rab11b & 2F9L & $2 \mathrm{~F} 9 \mathrm{M}$ & $\mathrm{ND}^{\wedge}$ & ND & ND \\
\hline Rab13 & ND & ND & ND & ND & ND \\
\hline Rab14 & $\begin{array}{c}1 \mathrm{Z0F} \\
4 \mathrm{DRZ}\end{array}$ & ND & $\mathrm{ND}^{\wedge}$ & ND & ND \\
\hline Rab18 & ND & $1 \mathrm{X} 3 \mathrm{~S}$ & ND & ND & ND \\
\hline Rab24 & ND & ND & ND & ND & ND \\
\hline Rab26 & ND & $2 \mathrm{G} 6 \mathrm{~B}$ & ND & ND & ND \\
\hline Rab27a & ND & HUF6 & $\mathrm{ND}^{\wedge}$ & ND & ND \\
\hline Rab28 & $2 \mathrm{HXS}$ & $3 \mathrm{E} 5 \mathrm{H}$ & ND & ND & ND \\
\hline Rab35 & $\mathrm{ND}^{\#}$ & ND & $\mathrm{ND}^{\wedge}$ & $\mathrm{ND}^{\wedge}$ & ND \\
\hline Rab37 & ND & ND & ND & ND & ND \\
\hline \multicolumn{6}{|c|}{ Rho GTPases } \\
\hline RhoA & $\begin{array}{l}1 \mathrm{FTN} \\
5 \mathrm{E} 26 \\
6 \mathrm{KX} 2\end{array}$ & $\begin{array}{l}\text { 1A2B } \\
3 \text { TVD }\end{array}$ & $\mathrm{ND}^{\wedge}$ & $\mathrm{ND}^{\wedge}$ & ND \\
\hline $\mathrm{Cdc} 42$ & ND\# & 2QR2 & 1CEE (WASP) & $\mathrm{ND}^{\wedge}$ & $\mathrm{ND}^{\wedge}$ \\
\hline Rac1 & $\begin{array}{l}5 \mathrm{~N} 6 \mathrm{O} \\
\text { 6AGP }\end{array}$ & $\begin{array}{l}\text { 1MH1 } \\
\mathbf{3 T H 5}\end{array}$ & $\mathrm{ND}^{\wedge}$ & $\begin{array}{c}\text { 1FOE (TIAM1) } \\
\text { 4YON (P-Rex1) } \\
\text { 5O33 (Kalirin } \\
\text { DH1) }\end{array}$ & $\mathrm{ND}^{\wedge}$ \\
\hline
\end{tabular}




\begin{tabular}{|c|c|c|c|c|c|}
\hline TC10 & ND & 2ATX & $\begin{array}{l}\text { 2KE4 (CDc42- } \\
\text { interacting } \\
\text { domain of } \\
\text { CIP4) }\end{array}$ & ND & ND \\
\hline $\begin{array}{l}\text { RND3/ } \\
\text { RhoE }\end{array}$ & ND & $\begin{array}{c}\text { 1GWN (only } \\
\text { core domain) } \\
1 \mathrm{M} 7 \mathrm{~B}\end{array}$ & $\mathrm{ND}^{\wedge}$ & ND & ND \\
\hline \multicolumn{6}{|c|}{ Ras GTPases } \\
\hline RalA & $\begin{array}{l}1 \mathrm{U} 90 \\
1 \mathrm{U} 8 \mathrm{Z} \\
6 \mathrm{P} 0 \mathrm{O} \\
6 \mathrm{P} 0 \mathrm{~J}\end{array}$ & ND & $\begin{array}{l}\text { 1UAD (Sec5) } \\
\text { 1ZC3 (Exo84) } \\
\text { 1ZC4 (Exo84) }\end{array}$ & ND & ND \\
\hline (R) Rad & $\begin{array}{c}2 \mathrm{DPX} \\
2 \mathrm{GJS}\end{array}$ & $\begin{array}{l}\text { 3Q72 }(\mathrm{G} \\
\text { domain) } \\
\text { 3Q7P }(\mathrm{G} \\
\text { domain) }\end{array}$ & ND & ND & ND \\
\hline Rap1 & ND & ND & $\mathrm{ND}^{\wedge}$ & ND & ND \\
\hline H-Ras & 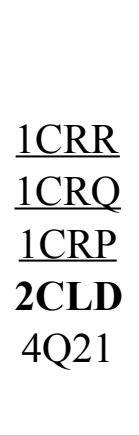 & $\begin{array}{c}\text { 121P } \\
1 \mathrm{CTQ} \\
1 \mathrm{PLK} \\
\text { 1QRA } \\
3 \mathrm{TGP} \\
4 \mathrm{RSG} \\
\text { 4EFL } \\
\text { 5P21 }\end{array}$ & $\mathrm{ND}^{\wedge}$ & $\begin{array}{c}\text { 1NVX (SOS) } \\
\text { 1NVW (SOS) } \\
\text { 1NVU (SOS) } \\
\text { 1BKD(SOS-1) } \\
\text { 4URY(SOS) }\end{array}$ & $\begin{array}{c}\text { 1WQ1 } \\
\text { (RasGAP) }\end{array}$ \\
\hline N-Ras & ND & $5 \mathrm{UHV}$ & ND & ND & ND \\
\hline
\end{tabular}




\begin{tabular}{|c|c|c|c|c|c|}
\hline K-Ras & $\begin{array}{l}4 \mathrm{OBE} \\
5 \mathrm{VQ} 8 \\
5 \mathrm{~W} 22 \\
6 \mathrm{MBT} \\
6 \mathrm{MBU}\end{array}$ & $\begin{array}{c}\text { 5VQ2 } \\
5 \mathrm{VQ6} \\
6 \mathrm{GOD} \\
6 \mathrm{VC} 8\end{array}$ & $\mathrm{ND}^{\wedge}$ & ND & ND \\
\hline
\end{tabular}

Structures identification codes (PDB ID codes) were assessed from the Protein Data Bank, http://www.pdb.org. As source organism taxonomy was selected Homo sapiens, Mus musculus, and Rattus norvegicus. X-ray and NMR structures are included. NMR structures are underlined. Only the structures of GTPases with a single $\mathrm{Mg}^{2+}$ were included. Structures in the complex with the effectors unrelated to diabetes were omitted. ND - not determined for GTPases; ND^ - there exist crystal structures of GTPaseeffector/GEF/GAP complexes, but unrelated to diabetes, insulin resistance or insulin-dependent tissue; ND\# - crystal structure of GDPbound form exists only in the in complex with GEF; \&GTP or its non-hydrolyzable analog, including GppNHp, GTPgammaS, GMPPCP; PDB ID codes in bold represent structures used for the generation of Figure 1. 
Supplementary Table 2 corresponding to Figure 3. List of crystal structures of the enzymes of mevalonate pathway playing a role in diabetes mellitus

\begin{tabular}{|c|c|c|c|}
\hline Enzyme & Only enzyme & With substrate/product & With inhibitor \\
\hline HMG-CoA reductase & $\mathrm{ND}^{\wedge}$ & $\begin{array}{c}\text { 1DQ8 (HMG and CoA) } \\
\text { 1DQ9 (HMG-CoA) } \\
\text { 1DQA (HMG, CoA and NADP+) }\end{array}$ & $\begin{array}{l}\text { 1HW9 (Simvastatin) } \\
\text { 1HWI (Fluvastatin) } \\
\text { 1HWK (Artovastatin) }\end{array}$ \\
\hline $\begin{array}{l}\text { Farnesyl } \\
\text { pyrophosphate } \\
\text { synthase (FPPS)/ } \\
\text { farnesyl diphosphate } \\
\text { synthase (FDPS) }\end{array}$ & $\begin{array}{c}1 \mathrm{ZW5} \\
2 \mathrm{~F} 7 \mathrm{M} \\
4 \mathrm{XQR} \\
4 \mathrm{XQS}\left(\mathrm{Mg}^{2+}\right) \\
4 \mathrm{XQT} \\
\left(3 \mathrm{Mg}^{2+}\right)\end{array}$ & 5JA0 (FPP) & $\begin{array}{l}\text { 2F89 (pamidronate) } \\
\text { 2F8C (zoledronate) } \\
\text { 2F92 (alendronate) }\end{array}$ \\
\hline $\begin{array}{l}\text { Geranylgeranyl } \\
\text { diphosphate synthase } \\
\text { (GGPPS) }\end{array}$ & $\mathrm{ND}^{\wedge}$ & 2Q80 (GGPP) & $\mathrm{ND}^{\wedge}$ \\
\hline $\begin{array}{l}\text { Farnesyltransferase } \\
\text { (FTase) }\end{array}$ & 1FT1 & $\begin{array}{c}\text { 1FPP (FPP) } \\
\left.\text { 1FT2 (FPP, Zn }{ }^{2+}\right) \\
\text { 1JC6 (FPP, TKCVFM peptide substrate) } \\
\text { 1KZP (farnesylated K-Ras4b) } \\
\text { 1TN8 (FPP, H-Ras) } \\
\text { 2H6F (farnesylated DDPTASACVLS peptide) }\end{array}$ & $\begin{array}{c}\text { 1SA4 (R115777) } \\
\text { 1SA5 (BMS-214662) }\end{array}$ \\
\hline
\end{tabular}




\begin{tabular}{|c|c|c|c|}
\hline $\begin{array}{l}\text { Geranylgeranyltransf } \\
\text { erase-I (GGTase-I) }\end{array}$ & 1DCE & $\begin{array}{c}\text { 1N4P (GRG) } \\
\text { 1N4Q (GGPP*, geranylgeranylated KKKSKTKCVIL peptide) } \\
\text { 1N4R (geranylgeranylated KKKSKTKCVIL peptide) } \\
\text { 1N4S (GGPP, geranylgeranylated KKKSKTKCVIL peptide) } \\
\text { 1TNO (GGPP*, geranylgeranylated KKKSKTKCVIM peptide } \\
\text { from K-Ras4b) } \\
\text { 1TNZ (GGPP*, geranylgeranylated RRCVLL peptide from } \\
\text { Cdc42) }\end{array}$ & $\mathrm{ND}^{\wedge}$ \\
\hline $\begin{array}{l}\text { Geranylgeranyltransf } \\
\text { erase type- } 2 \\
\text { (GGTase- } 2 \text { ) }\end{array}$ & 3DSS & $\begin{array}{c}\text { 3DST (GRG) } \\
\text { 3DSU (FPP, } \mathrm{Ca}^{2+} \mathrm{Zn}^{2+} \text { ) } \\
\text { 3DSV (monoprenylated SCS } \underline{\mathrm{C}} \text { peptide from Rab7) } \\
\text { 3DSW (monoprenylated SCSC peptide from Rab7) } \\
\text { 3DSX (diprenylated } \underline{\mathrm{SCSC}} \text { peptide from Rab7) }\end{array}$ & $\mathrm{ND}^{\wedge}$ \\
\hline $\begin{array}{l}\text { Geranylgeranyltransf } \\
\text { erase type-III } \\
\text { (GGTase-III) }\end{array}$ & $6 J 6 X^{\&}$ & $\mathrm{ND}^{\wedge}$ & $\mathrm{ND}^{\wedge}$ \\
\hline
\end{tabular}

Supplementary Table 2 corresponding to Figure 3. List of crystal structures of the enzymes of mevalonate pathway playing a role in diabetes mellitus.

Structures identification codes (PDB ID codes) were assessed from the Protein Data Bank, http://www.pdb.org. As source organism taxonomy was selected Homo sapiens, Mus musculus, and Rattus norvegicus. Structures in the complex with the inhibitors unrelated to diabetes were omitted. ND - not determined for the enzymes, $\mathrm{ND}^{\wedge}$ - there exist crystal structures of enzyme/substrate/product or 
enzyme-inhibitor complexes, but unrelated to diabetes, insulin resistance or insulin-dependent tissue, *analog, \& deposited, but unpublished. PDB ID codes in bold represent structures used for the generation of Figure 3. 